Article

\title{
Iron-Binding and Anti-Fenton Properties of Novel Amino Acid-Derived Cyclic Imide Dioximes
}

\author{
Janez Mravljak@ and Žiga Jakopin *(1) \\ Faculty of Pharmacy, University of Ljubljana, Aškerčeva 7, SI-1000 Ljubljana, Slovenia; \\ janez.mravljak@ffa.uni-lj.si \\ * Correspondence: ziga.jakopin@ffa.uni-lj.si; Tel.: +386-1476-9646
}

Received: 26 July 2019; Accepted: 8 October 2019; Published: 11 October 2019

check for updates

\begin{abstract}
We present a novel route for the preparation of amino acid-derived cyclic imide dioxime derivatives. Readily accessible amino acids were conveniently converted to their corresponding cyclic imide dioximes in simple synthetic steps. The aim of this work was to describe and compare the iron-chelating and antioxidant properties of synthesized compounds in relation to their molecular structure, and in particular, which of those features are essential for iron(II)-chelating ability. The glutarimide dioxime moiety has been established as an iron(II)-binding motif and imparts potent anti-Fenton properties to the compounds. Compound 3 was shown to strongly suppress hydroxyl radical formation by preventing iron cycling via Fe-complexation. These findings provide insights into the structural requirements for achieving anti-Fenton activity and highlight the potential use of glutarimide dioximes as antioxidants.
\end{abstract}

Keywords: amino acid; building block; iron(II)-chelating agents; cyclic imide dioxime; antioxidant; Fenton reaction; anti-Fenton

\section{Introduction}

Cyclic imide dioximes, such as glutarimide dioxime (I; also known as 2,6-dihydroxyiminopiperidine) [1] and succinimide dioxime (II; also known as 2,5-dihydroxyiminopyrrolidine) [2] (shown in Figure 1), have long been known for their ability to form complexes with metals [3-7]. Cyclic imide dioximes are commonly typified by a tridentate binding site [8] and have already been evaluated for their major transition metal chelating abilities [9,10]. I, for example, is able to bind Fe(III) and subsequently form a strong complex [3a]. The solved crystal structures of the complex revealed 1:1 and 1:2 metal-ligand complex with tridentate coordination in which the ligand coordinates to iron through the two oxime oxygen atoms and the imide nitrogen atom. The bond lengths of Fe-O and Fe-N as well as the high ionic charge and small radius of $\mathrm{Fe}(\mathrm{III})$ are of particular importance in determining the order of binding strength when compared to other tested metal ions. The binding strength of I with these ions follows the order: $\mathrm{Fe}(\mathrm{III})$ $>\mathrm{Cu}$ (II) $>\mathrm{Pb}$ (II) $>\mathrm{Ni}$ (II) [3]. It was suggested that if grafted onto a solid substrate, its complexing ability could be even greater [11]. Several imide dioxime derivatives, such as phthalimide dioxime and naphthalimide dioxime, have recently been described [10,12]. Interestingly, the imide dioxime functionality has also found use in the synthesis of pharmaceutically active compounds [13]. 


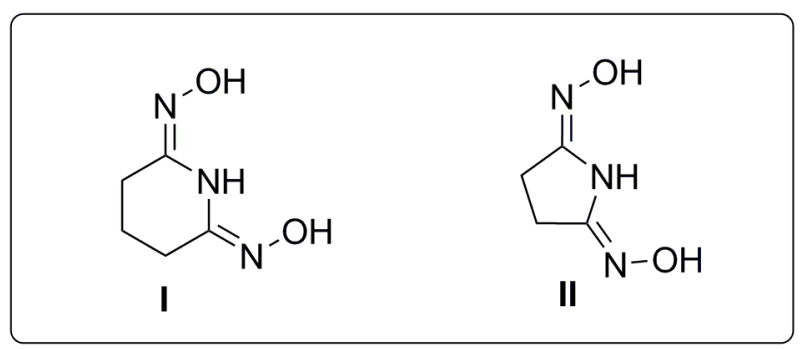

Figure 1. Basic cyclic imide dioxime scaffolds.

In biological systems, reactive oxygen species (ROS) often originate from the interaction of redox-active metal ions with superoxide and/or hydrogen peroxide (Fenton reaction) consequently leading to oxidative damage and contribute to neurodegenerative disorders like Alzheimer's and Parkinson's. Free iron (either as Fe(II) or Fe(III)) is therefore toxic even at very low concentrations [14]. Its chelation is also beneficial in cases of thalassemia to neutralise the iron overload in the body [15]. Given the fact that Fenton chemistry depends on the oxidation of redox-active metal ions in their reduced form (e.g., $\mathrm{Fe}(\mathrm{II})$ or $\mathrm{Cu}(\mathrm{I})$ ) with hydrogen peroxide to generate hydroxyl radicals, the redox chemistry of these elements is closely regulated. Thus, contrary to the common antioxidants, which inactivate ROS only after they have been formed, by the chelation of these metal ions with chelating agents, the formation of ROS could be prevented. For example, quercetin belongs to the flavonoid class of polyphenolic antioxidants whose antioxidant properties have been shown to rely mostly on preventing the Fenton chemistry via chelation, and the subsequent sequestration, of iron(II) [16]. Extensive research has been carried out in recent years on the design of iron chelators and pharmacophore conjugates affording molecules with multimodal functions (selected iron-binding motifs are depicted in Figure 2) [17].<smiles>Oc1ccccc1O</smiles>

catechol<smiles>O=c1ccccn1O</smiles>

1-hydroxypyridin-2(1H)-one<smiles>Oc1cccc2cccnc12</smiles>

8-hydroxyquinoline<smiles>O=c1ccocc1O</smiles>

3-hydroxy-4H-pyran-4-one<smiles>[R]N1CC(=O)NC(=O)C1</smiles>

2,6-piperidinedione

Figure 2. Several examples of different iron-binding motifs [14,16].

These findings prompted us to design novel imide dioximes with potential iron(II)-chelating abilities. In the search for a synthetic route to novel cyclic imide dioximes, we used readily accessible amino acids which were converted to the corresponding cyclic imide dioximes in simple synthetic steps. The designed and synthesized cyclic imide dioximes are amenable to integration into larger structures or onto resins, either directly or via a linker. The present study was conducted with a focus on the complexation of iron(II). The chelating effect of the synthesized compounds on ferrous ions was established by the ferrozine assay and compared to those of quercetin and ethylenediaminetetracetic acid (EDTA). The aim of this work was to describe and compare the iron-chelating and antioxidant properties of synthesized compounds in relation to their molecular structure, in particular, which of those features are essential for iron(II)-chelating ability. Finally, the selected compounds were screened 
for their anti-Fenton properties by determining their ability to halt copper and iron redox cycling via metal complexation in the ascorbate redox system assay.

\section{Materials and Methods}

\subsection{Preparation of Cyclic Imide Dioximes}

Chemicals were obtained from Acros, Sigma-Aldrich and TCI, and used without further purification. Succinimide dioxime and glutarimide dioxime were synthesized as previously described [3,18]. Analytical thin layer chromatography (TLC) was performed on Merck 60 F254 silica gel plates $(0.25 \mathrm{~mm})$, using visualization with ultraviolet light and ninhydrin. Column chromatography was carried out on silica gel 60 (particle size 240-400 mesh). Melting points were determined on a Reichert hot stage microscope and are uncorrected. ${ }^{1} \mathrm{H}$ - and ${ }^{13} \mathrm{C}-\mathrm{NMR}$ spectra were recorded at $400 \mathrm{MHz}$ and $100 \mathrm{MHz}$, respectively, on a Bruker AVANCE III spectrometer in DMSO-d $\mathrm{d}_{6}$ or $\mathrm{CDCl}_{3}$ solution with tetramethylsilane (TMS) as internal standard. Spectra were assigned using gradient correlation spectroscopy (COSY) and heteronuclear single quantum coherence (HSQC) spectroscopy experiments. IR spectra were recorded on a Perkin-Elmer 1600 FT-IR spectrometer. Mass spectra were obtained using a VG-Analytical Autospec $Q$ mass spectrometer. Microanalyses were performed on a $240{ }^{\circ} \mathrm{C}$ Perkin-Elmer $\mathrm{CHN}$ analyzer.

\subsubsection{Chemistry}

The synthesis of novel amino acid-derived cyclic imide dioximes has been carried out as shown in Schemes 1 and 2.
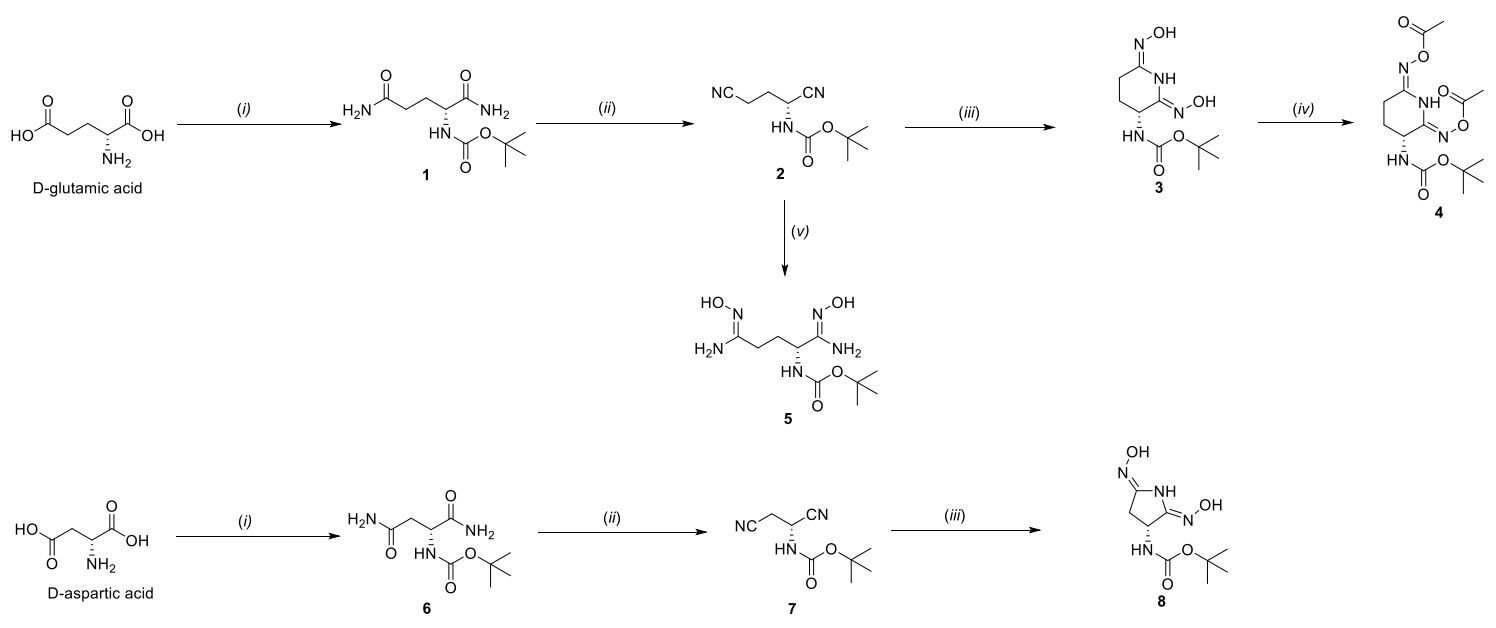

Scheme 1. Reagents and conditions: (i) (a) (Boc) $)_{2} \mathrm{O}, \mathrm{Na}_{2} \mathrm{CO}_{3}$, dioxan/water; (b) (Boc) ${ }_{2} \mathrm{O},\left(\mathrm{NH}_{4}\right)_{2} \mathrm{CO}_{3}$, pyridine, tetrahydrofuran, $\mathrm{rt}$; (ii) cyanuric chloride, $\mathrm{DMF}$, first at $0^{\circ} \mathrm{C}$ then at rt; (iii) $\mathrm{NH}_{2} \mathrm{OH} \times \mathrm{HCl}$, $\mathrm{Et}_{3} \mathrm{~N}$, EtOH, reflux; (iv) $\mathrm{AcCl}$, diisopropylethylamine, $\mathrm{CH}_{2} \mathrm{Cl}_{2}$, first at $0{ }^{\circ} \mathrm{C}$ then at $\mathrm{rt}$; (v) $\mathrm{NH}_{2} \mathrm{OH} \times$ $\mathrm{HCl}, \mathrm{Na}_{2} \mathrm{CO}_{3}, \mathrm{EtOH}, \mathrm{H}_{2} \mathrm{O}$, rt.

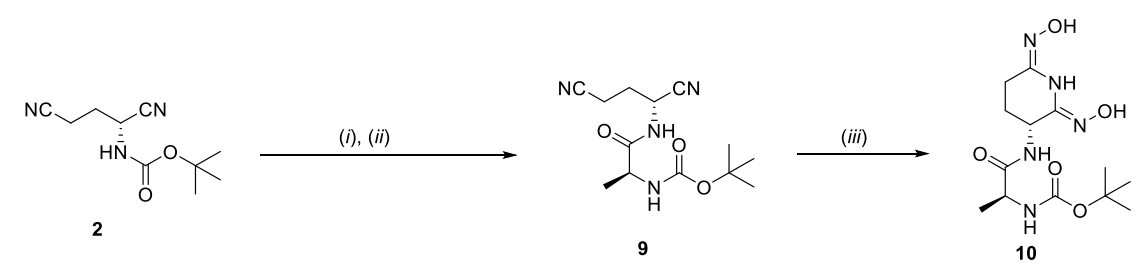

Scheme 2. Reagents and conditions: (i) TFA/ $\mathrm{CH}_{2} \mathrm{Cl}_{2}$ (1:5); (ii) Boc-L-Ala, TBTU, diisopropylethylamine, $\mathrm{CH}_{2} \mathrm{Cl}_{2}$; (iii) $\mathrm{NH}_{2} \mathrm{OH} \times \mathrm{HCl}, \mathrm{Et}_{3} \mathrm{~N}, \mathrm{EtOH}$, reflux. 


\section{Conversion of Amides to Nitriles}

To a stirred solution of amide $(1.0 \mathrm{mmol})$ in dry DMF $(5 \mathrm{~mL})$, cyanuric chloride $(1 \mathrm{mmol})$ was added at $0{ }^{\circ} \mathrm{C}$. After stirring for 15 minthe mixture was allowed to warm to room temperature and stirring then continued for $3 \mathrm{~h}$. The reaction mixture was quenched with water $(30 \mathrm{~mL})$ and extracted with ethyl acetate $(3 \times 50 \mathrm{~mL})$. The organic phase was washed with $10 \%$ citric acid $(2 \times 10 \mathrm{~mL})$, water $(20 \mathrm{~mL})$, saturated $\mathrm{NaHCO}_{3}$ solution $(2 \times 10 \mathrm{~mL})$, water $(20 \mathrm{~mL})$ and then dried over anhydrous $\mathrm{Na}_{2} \mathrm{SO}_{4}$. The solvent was removed under reduced pressure and the residue was additionally purified by flash chromatography on a silica gel column using chloroform/methanol (20:1) as eluant to afford the corresponding nitrile.

\section{Synthesis of Cyclic Imide Dioximes}

To a stirred solution of nitrile $(1.0 \mathrm{mmol})$ and hydroxylamine hydrochloride $(2.5 \mathrm{mmol})$ in ethanol, triethylamine $(1.5 \mathrm{mmol})$ was added. The mixture was then heated to reflux and stirred overnight. The solvent was removed under reduced pressure and the residue was additionally purified by flash chromatography on a silica gel column if necessary using chloroform/methanol (9:1) as eluant to afford the corresponding amidoxime.

\section{General Procedure for Bisamidoxime Synthesis}

To a stirred solution of nitrile $(1.0 \mathrm{mmol})$ and hydroxylamine hydrochloride $(2.5 \mathrm{mmol})$ in a mixture of ethanol and water, sodium carbonate $(2.5 \mathrm{mmol})$ was added. The mixture was then stirred overnight at room temperature. The solvent was removed under reduced pressure and the residue was additionally purified by flash chromatography on a silica gel column if necessary using chloroform/methanol (9:1) as eluant to afford the corresponding amidoxime.

\subsubsection{Characterization of Compounds}

Tert-butyl (1R)-(1,5-diamino-1,5-dioxopentan-2-yl)carbamate (1)

White amorphous powder, yield: 79\%; m.p. $128-131{ }^{\circ} \mathrm{C} .{ }^{1} \mathrm{H}-\mathrm{NMR}\left(\mathrm{DMSO}-\mathrm{d}_{6}, 400 \mathrm{MHz}\right): \delta=1.38$ (s, 9H, 3CH $\left.\mathrm{CH}_{3}\right), 1.62-1.72\left(\mathrm{~m}, 1 \mathrm{H}, \mathrm{CH}_{2 \mathrm{~A}}-\beta-\mathrm{Glu}\right), 1.78-1.87\left(\mathrm{~m}, 1 \mathrm{H}, \mathrm{CH}_{2 \mathrm{~B}}-\beta-\mathrm{Glu}\right), 2.08(\mathrm{t}, 2 \mathrm{H}, \mathrm{J}=6.8 \mathrm{~Hz}$, $\left.\mathrm{CH}_{2}-\gamma-\mathrm{Glu}\right), 3.79-3.83$ (m, 1H, CH-Ala), 6.76-6.78 (m, 2H, $\left.\mathrm{CONH}_{2}\right), 6.98\left(\mathrm{~s}, 1 \mathrm{H}, \mathrm{CONH}_{2 \mathrm{~A}}\right), 7.24-7.28$ $\left(\mathrm{m}, 2 \mathrm{H}, \mathrm{CONH}_{2 \mathrm{~B}}\right.$ and $\left.\mathrm{NHBoc}\right)$ ppm.

Tert-butyl (1R)-1,3-dicyanopropylcarbamate (2)

Synthesized according to the General procedure for the conversion of amides to nitriles. White amorphous powder, yield: 87\%; m.p. $93-94{ }^{\circ} \mathrm{C} .1 \mathrm{H}-\mathrm{NMR}$ (DMSO-d $6,400 \mathrm{MHz}$ ): $\delta=1.41\left(\mathrm{~s}, 9 \mathrm{H}, 3 \mathrm{CH}_{3}\right), 2.00-2.11\left(\mathrm{~m}, 2 \mathrm{H}, \mathrm{CH}_{2}-\beta-\mathrm{Glu}\right), 2.59\left(\mathrm{t}, 2 \mathrm{H}, J=7.2 \mathrm{~Hz}, \mathrm{CH}_{2}-\gamma-\mathrm{Glu}\right)$, 4.49-4.56 (m, 1H, CH), $7.80(\mathrm{~d}, 1 \mathrm{H}, J=6.0 \mathrm{~Hz}, \mathrm{NH})$ ppm. MS (ESI): $\mathrm{m} / z(\%)=210.1$ $(\mathrm{M}+\mathrm{H})^{+} . \mathrm{IR}(\mathrm{KBr}): v=3358,2984,2941,2258,1685,1517,1372,1340,1311,1280,1158,1057,875,604 \mathrm{~cm}^{-1}$. HRMS Calcd for $\mathrm{C}_{10} \mathrm{H}_{16} \mathrm{~N}_{3} \mathrm{O}_{2} \mathrm{~m} / z$ : $210.1250(\mathrm{M}+\mathrm{H})^{+}$, found 210.1243. Anal. Calcd for $\mathrm{C}_{10} \mathrm{H}_{15} \mathrm{~N}_{3} \mathrm{O}_{2}$ (\%): C, 57.40; H, 7.23; N, 20.08. Found: C, 57.38; H, 7.40; N, 19.94. $[\alpha]_{\mathrm{D} 20}=+102.6^{\circ}\left(c 0.12, \mathrm{CH}_{2} \mathrm{Cl}_{2}\right)$.

Tert-butyl (3R)-2,6-bis(hydroxyimino)piperidinylcarbamate (3)

Synthesized according to the General procedure for the synthesis of cyclic imide dioximes. Beige amorphous powder, yield: 83\%; m.p. $189-193{ }^{\circ} \mathrm{C} .{ }^{1} \mathrm{H}-\mathrm{NMR}$ (DMSO-d $\left.6,400 \mathrm{MHz}\right): \delta=1.39$ $\left(\mathrm{s}, 9 \mathrm{H}, 3 \mathrm{CH}_{3}\right), 1.67-1.78\left(\mathrm{~m}, 1 \mathrm{H}, \mathrm{CH}_{2 \mathrm{~A}}-\beta\right), 1.82-1.88\left(\mathrm{~m}, 1 \mathrm{H}, \mathrm{CH}_{2 \mathrm{~B}}-\beta\right), 2.41-2.44\left(\mathrm{~m}, 2 \mathrm{H}, \mathrm{CH}_{2}-\gamma\right), 4.37-4.38$ $(\mathrm{m}, 1 \mathrm{H}, \mathrm{CH}), 7.19(\mathrm{~d}, 1 \mathrm{H}, J=8.8 \mathrm{~Hz}, \mathrm{Boc}-\mathrm{NH}), 8.29(\mathrm{~s}, 1 \mathrm{H}, \mathrm{NH}), 9.94(\mathrm{~s}, 1 \mathrm{H}, \mathrm{OH}), 10.28(\mathrm{~s}, 1 \mathrm{H}, \mathrm{OH})$

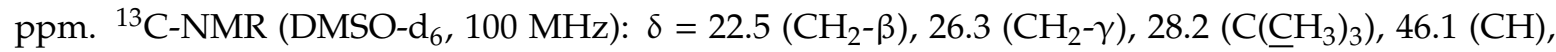
$77.9\left(\underline{\mathrm{C}}\left(\mathrm{CH}_{3}\right)_{3}\right), 143.9(\mathrm{C}=\mathrm{N}), 144.8(\mathrm{C}=\mathrm{N}), 155.0(\mathrm{CO})$ ppm. MS (ESI): $m / z(\%)=259.1(\mathrm{M}+\mathrm{H})^{+} . \mathrm{IR}(\mathrm{KBr})$ : $v=3401,3349,2981,2932,2363,1662,1521,1391,1312,1246,1166,1050,1022,982,960,927,855$, 
$779 \mathrm{~cm}^{-1}$. HRMS Calcd for $\mathrm{C}_{10} \mathrm{H}_{19} \mathrm{~N}_{4} \mathrm{O}_{4} \mathrm{~m} / z: 259.1406(\mathrm{M}+\mathrm{H})^{+}$, found 259.1398. Anal. Calcd for $\mathrm{C}_{10} \mathrm{H}_{18} \mathrm{~N}_{4} \mathrm{O}_{4}(\%)$ : C, 46.50; H, 7.02; N, 21.69. Found: C, 46.78; H, 6.80; N, 21.28. $[\alpha]_{\mathrm{D}}^{20}=+5.7^{\circ}$ (c $0.16, \mathrm{CH}_{2} \mathrm{Cl}_{2}$ ).

Tert-butyl ((R,2Z,6Z)-2,6-bis(acetoxyimino)piperidin-3-yl)carbamate (4)

Synthesized according to the General procedure for the acetylation. Light yellow oil, yield: $71 \%$; ${ }^{1} \mathrm{H}-\mathrm{NMR}\left(\mathrm{CDCl}_{3}, 400 \mathrm{MHz}\right): \delta=1.42\left(\mathrm{~s}, 9 \mathrm{H}, 3 \mathrm{CH}_{3}\right), 1.81-1.91\left(\mathrm{~m}, 1 \mathrm{H}, \mathrm{CH}_{2}-\beta\right), 2.13-2.16\left(\mathrm{~m}, 1 \mathrm{H}, \mathrm{CH}_{2}-\beta\right)$, $2.19\left(\mathrm{~s}, 3 \mathrm{H}, \mathrm{CH}_{3} \mathrm{CO}\right), 2.21$ (s, 3H, $\left.\mathrm{CH}_{3} \mathrm{CO}\right), 2.57-2.66$ and $2.77-2.83\left(2 \mathrm{~m}, 1 \mathrm{H}\right.$ each, $\left.\mathrm{CH}_{2}-\gamma\right), 4.49-4.54$ $(\mathrm{m}, 1 \mathrm{H}, \mathrm{CH}), 5.33\left(\mathrm{~d}, 1 \mathrm{H}, J=6.4 \mathrm{~Hz}\right.$, Boc-NH), $8.34(\mathrm{~s}, 1 \mathrm{H}, \mathrm{NH}) \mathrm{ppm} .{ }^{13} \mathrm{C}-\mathrm{NMR}\left(\mathrm{CDCl}_{3}, 100 \mathrm{MHz}\right)$ : $\delta=19.5\left(2 \times \mathrm{CH}_{3}\right), 23.4\left(\mathrm{CH}_{2}-\beta\right), 26.0\left(\mathrm{CH}_{2}-\gamma\right), 28.3\left(\mathrm{C}\left(\underline{\mathrm{CH}}_{3}\right)_{3}\right), 30.9,48.0(\mathrm{CH}), 80.6\left(\underline{\mathrm{C}}\left(\mathrm{CH}_{3}\right)_{3}\right), 148.5$ $(\mathrm{C}=\mathrm{N}), 149.0(\mathrm{C}=\mathrm{N}), 155.1(\mathrm{CO}), 167.2(2 \times \mathrm{CO}) \mathrm{ppm}$. MS (ESI): $\mathrm{m} / z(\%)=343.2(\mathrm{M}+\mathrm{H})^{+}$. IR $(\mathrm{KBr})$ : $v=3313,2975,1762,1646,1518,1368,1205,1163,1059,1006,921,852 \mathrm{~cm}^{-1}$. HRMS Calcd for $\mathrm{C}_{14} \mathrm{H}_{23} \mathrm{~N}_{4} \mathrm{O}_{6} \mathrm{~m} / z: 343.1618(\mathrm{M}+\mathrm{H})^{+}$, found 343.1631. Anal. Calcd for $\mathrm{C}_{14} \mathrm{H}_{22} \mathrm{~N}_{4} \mathrm{O}_{6}(\%): C, 49.12$; $\mathrm{H}, 6.48 ; \mathrm{N}, 16.37$. Found: $\mathrm{C}, 49.40 ; \mathrm{H}, 6.43 ; \mathrm{N}, 16.00 .[\alpha]_{\mathrm{D}}{ }^{20}=+0.2^{\circ}(c 0.18, \mathrm{MeOH})$.

Tert-butyl ((R,1Z,5Z)-1,5-diamino-1,5-bis(hydroxyimino)pentan-2-yl)carbamate (5)

Synthesized according to the General procedure for the synthesis of bisamidoximes. Beige amorphous powder, yield: $74 \%$; m.p. $126-130{ }^{\circ} \mathrm{C} .{ }^{1} \mathrm{H}-\mathrm{NMR}\left(\mathrm{DMSO}_{6}, 400 \mathrm{MHz}\right): \delta=1.38\left(\mathrm{~s}, 9 \mathrm{H}, 3 \mathrm{CH}_{3}\right)$, 1.69-1.79 (m, 2H, $\left.\mathrm{CH}_{2}-\beta-\mathrm{Glu}\right), 1.89-1.91$ (m, 2H, $\left.\mathrm{CH}_{2}-\gamma-\mathrm{Glu}\right), 3.89$ (q, $\left.1 \mathrm{H}, J=8.0 \mathrm{~Hz}, \mathrm{CH}\right), 5.25$ (s, 2H, NH $\mathrm{NH}_{2}, 5.33\left(\mathrm{~s}, 2 \mathrm{H}, \mathrm{NH}_{2}\right), 6.80(\mathrm{~d}, 1 \mathrm{H}, J=9.2 \mathrm{~Hz}, \mathrm{Boc}-\mathrm{NH}), 8.73(\mathrm{~s}, 1 \mathrm{H}, \mathrm{OH}), 8.96(\mathrm{~s}, 1 \mathrm{H}, \mathrm{OH})$ ppm. ${ }^{13} \mathrm{C}-\mathrm{NMR}\left(\mathrm{DMSO}_{6}, 100 \mathrm{MHz}\right): \delta=28.1\left(\mathrm{CH}_{2}-\beta\right), 28.7\left(\mathrm{C}\left(\mathrm{CH}_{3}\right)_{3}\right), 30.8\left(\mathrm{CH}_{2}-\gamma\right), 51.6(\mathrm{CH})$, $78.5\left(\underline{\mathrm{C}}\left(\mathrm{CH}_{3}\right)_{3}\right), 152.8(\mathrm{C}=\mathrm{N}), 153.3(\mathrm{C}=\mathrm{N}), 155.3(\mathrm{CO}) \mathrm{ppm}$. MS (ESI): $m / z(\%)=276.2(\mathrm{M}+\mathrm{H})^{+} . \mathrm{IR}(\mathrm{KBr})$ : $v=3480,3365,2978,2368,1675,1521,1363,1248,1172,1046,949,922,834,702 \mathrm{~cm}^{-1}$. HRMS Calcd for $\mathrm{C}_{10} \mathrm{H}_{22} \mathrm{~N}_{5} \mathrm{O}_{4} \mathrm{~m} / z$ : $276.1672(\mathrm{M}+\mathrm{H})^{+}$, found 276.1664. Anal. Calcd for $\mathrm{C}_{10} \mathrm{H}_{21} \mathrm{~N}_{5} \mathrm{O}_{4}(\%): \mathrm{C}, 43.63$; $\mathrm{H}, 7.69 ; \mathrm{N}, 25.44$. Found: $\mathrm{C}, 43.91 ; \mathrm{H}, 7.82 ; \mathrm{N}, 25.38 .[\alpha]_{\mathrm{D}}{ }^{20}=+2.1^{\circ}\left(c 0.13, \mathrm{CH}_{2} \mathrm{Cl}_{2}\right)$.

Tert-butyl (1R)-3-amino-1-(aminocarbonyl)-3-oxopropylcarbamate (6)

White amorphous powder, yield: 85\%; m.p. $190-192{ }^{\circ} \mathrm{C} .{ }^{1} \mathrm{H}-\mathrm{NMR}\left(\mathrm{DMSO}-\mathrm{d}_{6}, 400 \mathrm{MHz}\right.$ ): $\delta=1.38$ (s, 9H, 3CH $\left.\mathrm{CH}_{3}\right), 2.31-2.43\left(\mathrm{~m}, 2 \mathrm{H}, \mathrm{CH}_{2}-\beta-\mathrm{Asp}\right), 4.13-4.19(\mathrm{~m}, 1 \mathrm{H}, \mathrm{CH}), 6.80(\mathrm{~d}, 1 \mathrm{H}, J=8.8 \mathrm{~Hz}, \mathrm{NH})$, 6.89 (s, 1H, CONH), 6.99 (s, 1H, CONH), 7.18 (s, 1H, CONH), 7.27 (s, 1H, CONH) ppm. MS (ESI): m/z $(\%)=232.1(\mathrm{M}+\mathrm{H})^{+} . \mathrm{IR}(\mathrm{KBr}): v=3392,3341,3196,2983,2936,2364,1525,1408,1368,1327,1275$, $1248,1175,1057,1029,950,858,814,770 \mathrm{~cm}^{-1}$. HRMS Calcd for $\mathrm{C}_{9} \mathrm{H}_{18} \mathrm{~N}_{3} \mathrm{O}_{4} \mathrm{~m} / z: 232.1297(\mathrm{M}+\mathrm{H})^{+}$, found 232.1298. $[\alpha]_{\mathrm{D}}{ }^{20}=+8.0^{\circ}\left(c 0.16, \mathrm{CH}_{2} \mathrm{Cl}_{2}\right)$.

Tert-butyl (1R)-1,2-dicyanoethylcarbamate (7)

Synthesized according to the General procedure for the conversion of amides to nitriles. White amorphous powder, yield: $76 \%$; m.p. $133-136{ }^{\circ} \mathrm{C} .{ }^{1} \mathrm{H}-\mathrm{NMR}\left(\mathrm{DMSO}-\mathrm{d}_{6}, 400 \mathrm{MHz}\right): \delta=1.43$ (s, 9H, 3CH 3 ), 3.11-3.14 (m, 2H, $\left.\mathrm{CH}_{2}-\beta-\mathrm{Asp}\right), 4.91-4.96(\mathrm{~m}, 1 \mathrm{H}, \mathrm{CH}), 8.11(\mathrm{~d}, 1 \mathrm{H}, J=8.0 \mathrm{~Hz}, \mathrm{NH}) \mathrm{ppm}$. MS (ESI): $m / z(\%)=194.1(\mathrm{M}-\mathrm{H})^{-}$. IR (KBr): $v=3358,2984,2941,2258,1685,1517,1372,1340,1311$, 1280, 1158, 1057, 875, $604 \mathrm{~cm}^{-1}$. HRMS Calcd for $\mathrm{C}_{9} \mathrm{H}_{12} \mathrm{~N}_{3} \mathrm{O}_{2} \mathrm{~m} / \mathrm{z}: 194.0930(\mathrm{M}-\mathrm{H})^{-}$, found 194.0933. Anal. Calcd for $\mathrm{C}_{9} \mathrm{H}_{13} \mathrm{~N}_{3} \mathrm{O}_{2} \times 0.15 \mathrm{H}_{2} \mathrm{O}(\%)$ : C, 54.62; H, 6.77; N, 21.23. Found: C, 54.61; $\mathrm{H}, 6.79$; $\mathrm{N}, 21.22 .[\alpha]_{\mathrm{D}}^{20}=+31.1^{\circ}\left(c 0.20, \mathrm{CH}_{2} \mathrm{Cl}_{2}\right)$.

Tert-butyl (3R)-2,5-bis(hydroxyimino)pyrrolidinylcarbamate (8)

Synthesized according to the General procedure for the synthesis of cyclic imide dioximes. Beige amorphous powder, yield: 93\%; m.p. $223-226{ }^{\circ} \mathrm{C} .{ }^{1} \mathrm{H}-\mathrm{NMR}\left(\mathrm{DMSO}-\mathrm{d}_{6}, 400 \mathrm{MHz}\right): \delta=1.38$ (s, 9H, 3CH $\left.\mathrm{CH}_{3}\right), 2.40-2.45$ (m, 1H, $\left.\mathrm{CH}_{2 \mathrm{~A}}-\beta-\mathrm{Asp}\right), 2.85-2.92\left(\mathrm{~m}, 1 \mathrm{H}, \mathrm{CH}_{2 \mathrm{~B}}-\beta-\mathrm{Asp}\right), 4.64-4.70(\mathrm{~m}, 1 \mathrm{H}$, $\mathrm{CH}), 7.35(\mathrm{~d}, 1 \mathrm{H}, J=8.8 \mathrm{~Hz}, \mathrm{Boc}-\mathrm{NH}), 8.18(\mathrm{~s}, 1 \mathrm{H}, \mathrm{NH}), 9.68(\mathrm{~s}, 1 \mathrm{H}, \mathrm{OH}), 9.87(\mathrm{~s}, 1 \mathrm{H}, \mathrm{OH}) \mathrm{ppm}$.

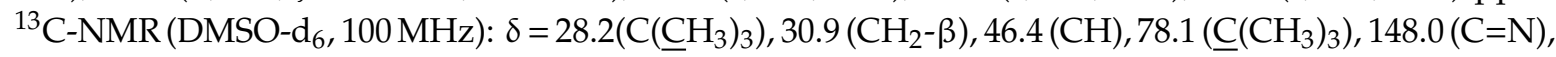


$150.4(\mathrm{C}=\mathrm{N}), 154.9(\mathrm{CO}) \mathrm{ppm}$. MS (ESI): $m / z(\%)=245.1(\mathrm{M}+\mathrm{H})^{+} . \mathrm{IR}(\mathrm{KBr}): v=3449,3349,3203,2883$, $2362,1683,1524,1370,1318,1273,1253,1167,1061,969,939,847,790,716 \mathrm{~cm}^{-1}$. HRMS Calcd for $\mathrm{C}_{9} \mathrm{H}_{17} \mathrm{~N}_{4} \mathrm{O}_{4} m / z: 245.1219(\mathrm{M}+\mathrm{H})^{+}$, found 245.1213. Anal. Calcd for $\mathrm{C}_{9} \mathrm{H}_{16} \mathrm{~N}_{4} \mathrm{O}_{4} \times 0.1 \mathrm{H}_{2} \mathrm{O}(\%): \mathrm{C}$, 43.93; $\mathrm{H}, 6.64 ; \mathrm{N}, 22.77$. Found: $\mathrm{C}, 44.16 ; \mathrm{H}, 6.41 ; \mathrm{N}, 22.49 .[\alpha]_{\mathrm{D}}{ }^{20}=+3.7^{\circ}\left(c 0.14, \mathrm{CH}_{2} \mathrm{Cl}_{2}\right)$.

Tert-butyl (1S)-2-\{[(1R)-1,3-dicyanopropyl]amino\}-1-methyl-2-oxoethylcarbamate (9)

To an ice-chilled stirred mixture of trifluoroacetic acid and dichloromethane $(v / v 1 / 5,5 \mathrm{~mL})$ Boc-protected compound $(0.5 \mathrm{mmol}) 2$ was added and the mixture allowed to warm to room temperature. After 3 hours the reaction was completed and the solvent was evaporated in vacuo. The residue was washed three times with diethyl ether giving sufficiently pure free amine which was dissolved in dichloromethane $(2 \mathrm{~mL})$. In a parallel reaction, to a stirred solution of Boc-L-Ala $(0.5 \mathrm{mmol})$ in dry dichloromethane $(10 \mathrm{~mL})$, diisopropylethylamine (DIPEA) $(1.50 \mathrm{mmol})$ and TBTU $(0.55 \mathrm{mmol})$ were added. After stirring for 45 minutes the solution of free amine in dichloromethane was added at $0{ }^{\circ} \mathrm{C}$, and the mixture was allowed to warm to room temperature and stirring then continued for $48 \mathrm{~h}$. Upon completion, the reaction mixture was diluted with dichloromethane $(30 \mathrm{~mL})$ and then washed with $1 \mathrm{M} \mathrm{HCl}(2 \times 10 \mathrm{~mL})$, water $(10 \mathrm{~mL})$, saturated $\mathrm{NaHCO}_{3}$ solution $(2 \times 10 \mathrm{~mL})$, water $(10 \mathrm{~mL})$ and then dried over anhydrous $\mathrm{Na}_{2} \mathrm{SO}_{4}$. The solvent was concentrated in vacuo and the residue was purified by flash silica gel column chromatography (gradient elution; starting eluent: chloroform/methanol 20:1 v/v) to afford compound 9. Yellow oil, yield: $57 \%$; ${ }^{1} \mathrm{H}-\mathrm{NMR}\left(\mathrm{CDCl}_{3}\right.$, $400 \mathrm{MHz}): \delta=1.39\left(\mathrm{t}, 3 \mathrm{H}, J=7.2 \mathrm{~Hz}, \mathrm{CH}_{3}-\mathrm{Ala}\right), 1.44\left(\mathrm{~s}, 9 \mathrm{H}, 3 \mathrm{CH}_{3}\right), 2.16-2.31\left(\mathrm{~m}, 2 \mathrm{H}, \mathrm{CH}_{2}-\beta-\mathrm{Glu}\right)$, 2.55 (t, 2H, $\left.J=7.2 \mathrm{~Hz}, \mathrm{CH}_{2}-\gamma-\mathrm{Glu}\right), 4.09-4.18$ (m, 1H, CH-Ala), 4.94-5.05 (m, 2H, CH-Glu, NH-Ala), $7.45(\mathrm{~d}, 1 \mathrm{H}, J=8.4 \mathrm{~Hz}, \mathrm{NH}-\mathrm{Glu})$ ppm. ${ }^{13} \mathrm{C}-\mathrm{NMR}\left(\mathrm{CDCl}_{3}, 100 \mathrm{MHz}\right): \delta=13.7\left(\mathrm{CH}_{2}-\gamma\right), 17.5\left(\underline{\mathrm{CH}} \mathrm{CH}_{3}\right)$,

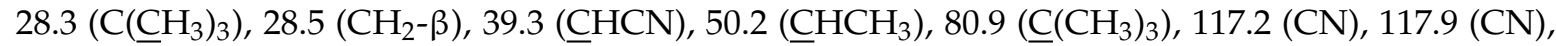
$156.0(\mathrm{CO}), 173.2(\mathrm{CO}) \mathrm{ppm}$. MS (ESI): $\mathrm{m} / \mathrm{z}(\%)=279.1(\mathrm{M}-\mathrm{H})^{-}$. HRMS Calcd for $\mathrm{C}_{13} \mathrm{H}_{19} \mathrm{~N}_{4} \mathrm{O}_{3} \mathrm{~m} / \mathrm{z}$ : 279.1457 (M-H) ${ }^{-}$, found 279.1454. Anal. Calcd for $\mathrm{C}_{13} \mathrm{H}_{20} \mathrm{~N}_{4} \mathrm{O}_{3}(\%)$ : C, 55.70; H, 7.19; N, 19.99 . Found: $\mathrm{C}, 55.26 ; \mathrm{H}, 6.80 ; \mathrm{N}, 20.16 .[\alpha]_{\mathrm{D}}{ }^{20}=-3.0^{\circ}\left(c 0.14, \mathrm{CH}_{2} \mathrm{Cl}_{2}\right)$.

Tert-butyl ((S)-1-(((R,Z)-6-(hydroxyamino)-2-(hydroxyimino)-2,3,4,5-tetrahydropyridin-3-yl)amino) -1-oxopropan-2-yl)carbamate (10)

Synthesized according to the General procedure for the synthesis of cyclic imide dioximes. Colorless oil, yield: $68 \% ;{ }^{1} \mathrm{H}-\mathrm{NMR}\left(\mathrm{DMSO}_{6}, 400 \mathrm{MHz}\right): \delta=1.18\left(\mathrm{t}, 3 \mathrm{H}, J=7.2 \mathrm{~Hz}, \mathrm{CH}_{3}\right.$-Ala), $1.37(\mathrm{~s}, 9 \mathrm{H}$, $\left.3 \mathrm{CH}_{3}\right), 1.68-1.76\left(\mathrm{~m}, 1 \mathrm{H}, \mathrm{CH}_{2 \mathrm{~A}}-\beta-\mathrm{Glu}\right), 1.77-1.87\left(\mathrm{~m}, 1 \mathrm{H}, \mathrm{CH}_{2 \mathrm{~B}}-\beta-\mathrm{Glu}\right), 3.96-3.98(\mathrm{~m}, 1 \mathrm{H}, \mathrm{CH}), 4.57-4.61$ $(\mathrm{m}, 1 \mathrm{H}, \mathrm{CH}), 6.83(\mathrm{~d}, 1 \mathrm{H}, J=7.6 \mathrm{~Hz}, \mathrm{Boc}-\mathrm{NH}), 8.26(\mathrm{~d}, 1 \mathrm{H}, J=8.0 \mathrm{~Hz}, \mathrm{CONH}), 8.34(\mathrm{~s}, 1 \mathrm{H}, \mathrm{NH})$, $10.00(\mathrm{~s}, 1 \mathrm{H}, \mathrm{OH}), 10.37(\mathrm{~s}, 1 \mathrm{H}, \mathrm{OH}) \mathrm{ppm} .{ }^{13} \mathrm{C}-\mathrm{NMR}(\mathrm{MeOD}, 100 \mathrm{MHz}): \delta=18.6\left(\underline{\mathrm{CH}}_{3} \mathrm{CH}\right), 23.3\left(\mathrm{CH}_{2}-\beta\right)$, $26.8\left(\mathrm{CH}_{2}-\gamma\right), 28.7\left(\mathrm{C}\left(\underline{\mathrm{CH}}_{3}\right)_{3}\right), 46.8\left(\underline{\mathrm{C}} \mathrm{HCH}_{3}\right), 51.8(\mathrm{CH}), 80.7\left(\underline{\mathrm{C}}\left(\mathrm{CH}_{3}\right)_{3}\right), 145.9(\mathrm{C}=\mathrm{N}), 146.5(\mathrm{C}=\mathrm{N})$, 157.6 (CO), 175.5 (CO) ppm. MS (ESI): $m / z(\%)=330.2(\mathrm{M}+\mathrm{H})^{+}$. IR (KBr): $v=3322,2980,2603,2496$, 1666, 1616, 1526, 1397, 1365, 1320, 1242, 1162, 1072, 1032, 980, $926 \mathrm{~cm}^{-1}$. HRMS Calcd for $\mathrm{C}_{13} \mathrm{H}_{23} \mathrm{~N}_{5} \mathrm{O}_{5}$ $m / z: 330.1777(\mathrm{M}+\mathrm{H})^{+}$, found 330.1780. Anal. Calcd for $\mathrm{C}_{13} \mathrm{H}_{23} \mathrm{~N}_{5} \mathrm{O}_{5}(\%): \mathrm{C}, 47.41 ; \mathrm{H}, 7.04 ; \mathrm{N}, 21.26$. Found: $\mathrm{C}, 47.16 ; \mathrm{H}, 6.81 ; \mathrm{N}, 21.49 .[\alpha]_{\mathrm{D}}{ }^{20}=-23.5^{\circ}(c 0.22, \mathrm{MeOH}) .{ }^{*} \mathrm{CH}_{2}-\gamma-\mathrm{Glu}$ hidden under the signal of DMSO.

\subsection{Antioxidant Evaluation}

\subsubsection{Ferrozine Assay}

The ferrous chelating ability of novel imide dioximes was evaluated by measuring the formation of the ferrous ion-ferrozine complex [19]. Solutions of samples $(15.7-500 \mu \mathrm{M})$ were prepared in a 1:1 $(v / v)$ mixture of methanol and potassium acetate buffer $(500 \mathrm{mM}, \mathrm{pH} 5.5)$. Freshly prepared water solution of $\mathrm{FeCl}_{2}(50 \mu \mathrm{L}, 250 \mu \mathrm{M})$ or water $(50 \mu \mathrm{L})$ was added to the $150 \mu \mathrm{L}$ of either solution of the test sample (15.7-500 $\mu \mathrm{M})$ or solvent (negative control) in a well. Quercetin and EDTA were used as positive control under the same assay conditions. Flat bottomed 96-well microtiter plate (TPP, 
Tissue Culture Test Plates) was shaken for $1 \mathrm{~min}$ and then left to stand at room temperature for $15 \mathrm{~min}$. Solution of ferrozine $(100 \mu \mathrm{L}, 1 \mathrm{mM})$ in potassium acetate buffer (500 mM, pH 5.5) was added in each well. Microtiter plate was than incubated at room temperature in the dark. After $30 \mathrm{~min}$, the absorbance was determined with a microplate reader (BioTek Synergy ${ }^{\mathrm{TM}}$ HT Multi-Detection Microplate Reader) at $562 \mathrm{~nm}$. Experiments were performed in triplicate. The percentage of inhibition of iron(II)-ferrozine chelating ability was calculated by using the following equation: Percentage of $I(\%)=\left(A_{0}-\left(A_{n}-\right.\right.$ $\left.\left.A_{b}\right)\right) / A_{0} \times 100$; where $A_{0}$ is the absorbance of negative control, $A_{n}$ is absorbance of the test sample and $\mathrm{A}_{\mathrm{b}}$ absorbance of the sample without $\mathrm{Fe}^{2+}$. Inhibition of iron(II)-ferrozine chelating ability was expressed as the concentration of test sample that chelates $50 \%$ of $\mathrm{Fe}^{2+}$ ions $\left(\mathrm{IC}_{50}\right) \pm$ standard deviation.

\subsubsection{Ascorbate Redox System Assay}

The ability of novel imide dioximes to halt copper and iron cycling was evaluated in ascorbate redox system assay as previously described [20]. All of the solutions, except $\mathrm{CuSO}_{4}$ and $\mathrm{FeCl}_{3}$ (dissolved and diluted in Milli-Q water only), were prepared in a phosphate (20 mM), NaCl (100 mM) buffer (PBS) at pH 7.4 with a final sample volume of $200 \mu \mathrm{L}$. Each experiment was performed in triplicate on flat bottomed 96-well microtiter plate (Greiner Bio-One). Hydroxyl radical production was measured as the conversion of coumarine-3-carboxylic acid (CCA) into 7-hydroxy-CCA $\left(\lambda_{\text {excitation }}=395 \mathrm{~nm}\right.$, $\left.\lambda_{\text {emission }}=450 \mathrm{~nm}\right)$. The general order of addition was as follows: CCA $(100 \mu \mathrm{M})$, ligand (compounds 3, I or II at $30 \mu \mathrm{M}$ or EDTA at $10 \mu \mathrm{M}$ in the case of iron-ascorbate studies), copper (10 $\mu \mathrm{M})$ or iron $(10 \mu \mathrm{M})$, and then ascorbate $(300 \mu \mathrm{M})$ after $30 \mathrm{~min}$ of incubation. All test solutions also contained $1 \mu \mathrm{M}$ desferryl.

\section{Results and Discussion}

\subsection{Synthesis}

Amino acids were transformed to their corresponding cyclic imide dioximes as shown in Scheme 1. They were first $N$-protected by reaction with Boc-anhydride, then converted in very good yields (79-85\% over two steps) to their corresponding diamides 1 [21] and 6, using the [( $\left.\left.\mathrm{Boc}_{2}\right) \mathrm{O}\right]-\left(\mathrm{NH}_{4}\right)_{2} \mathrm{CO}_{3}$ system [22]. In the following step, cyanuric chloride in dimethylformamide at room temperature was used as a dehydrating agent $[23,24]$ to convert the starting diamides quantitatively into their respective dinitriles 2 and 7. This two-step sequence for the transformation of $\alpha$-amino acids to $\alpha$-aminonitriles, although rarely encountered in the literature, is very convenient and affords products with high enantiomeric purity. Subsequent treatment of the dinitriles 2 and 7 with hydroxylamine hydrochloride in the presence of triethylamine in refluxing ethanol [24,25], yielded the corresponding cyclic imide dioximes 3 and 8 in excellent yields (83-93\%). A previously published report showed unambiguously that racemization, under the reaction conditions used for cyclization, does not take place [13]. Thus, we concluded that the stereochemistry of the starting amino acids was not compromised. Moreover, the obtained ${ }^{1} \mathrm{H}$ and ${ }^{13} \mathrm{C}$ NMR data were compared to the reported NMR data of the basic cyclic imide dioxime scaffolds I and II. The comparison has shown that the preferred tautomeric forms of $\mathbf{3}$ and $\mathbf{8}$ are those depicted in Scheme 1. The imide dioxime $\mathbf{3}$ was then treated with acetyl chloride affording the diacetylated product, compound 4 . Further, treatment of dinitrile 2 with hydroxylamine hydrochloride in an ethanol/water mixture, in the presence of sodium carbonate at room temperature [26], yielded bisamidoxime 5 in a good yield.

Next, we demonstrated that cyclic imide dioximes can be integrated into larger structures such as dipeptides (shown in Scheme 2). Thus, 2 underwent acidolytic removal of the N-Boc group, using a standard TFA- $\mathrm{CH}_{2} \mathrm{Cl}_{2}$ (1:5) protocol, yielding the free amine that immediately underwent TBTU-mediated coupling with Boc-L-Ala to afford compound 9. It was subsequently treated with hydroxylamine hydrochloride in the presence of triethylamine in refluxing ethanol to afford Compound 10, which consists of a glutarimide dioxime moiety linked to L-Ala. All the structures were fully characterized by spectroscopic data. 


\subsection{Evaluation of Iron-Chelating Properties}

The compounds were first evaluated for their iron(II)-chelating abilities in the ferrozine assay and compared to that of quercetin, a known iron(II)-chelating agent $\left(\mathrm{IC}_{50}=129 \pm 15 \mu \mathrm{M}\right)$ [14]. Ferrozine can quantitatively form complexes with iron(II); however, in the presence of chelating agents, the complex formation is disrupted, which can be measured spectrophotometrically $[19,27,28]$. The results obtained provided an insight into the structural requirements for iron(II) chelation of our set of compounds (see Table 1).

Table 1. Iron(II)-chelating properties of the synthesized compounds.

\begin{tabular}{cc}
\hline Compound & IC $_{\mathbf{5 0}}$ (Ferrozine Assay) \pm S.D. $[\boldsymbol{\mu M}]$ \\
\hline $\mathbf{3}$ & $268 \pm 37.7$ \\
$\mathbf{4}$ & $375 \pm 33.4$ \\
$\mathbf{5}$ & $671 \pm 30.7$ \\
$\mathbf{8}$ & $/$ \\
$\mathbf{1 0}$ & $895 \pm 33.1$ \\
$\mathbf{I}$ & $601 \pm 12.6$ \\
$\mathbf{I I}$ & $/$ \\
Quercetin & $129 \pm 15.0$ \\
EDTA & $21.1 \pm 0.7$ \\
\hline
\end{tabular}

Compound $3\left(\mathrm{IC}_{50}=268 \pm 37.7 \mu \mathrm{M}\right)$ proved as the most potent of the series, its iron(II)-chelating ability being only slightly lower than that of quercetin $\left(\mathrm{IC}_{50}=129 \pm 15.0 \mu \mathrm{M}\right)$. Evidently, the 6-membered glutarimide dioxime is pivotal for iron(II)-chelating properties (compounds $\mathbf{I}\left(\mathrm{IC}_{50}=601\right.$ $\pm 12.6 \mu \mathrm{M}), 3\left(\mathrm{IC}_{50}=268 \pm 37.7 \mu \mathrm{M}\right)$, and $4\left(\mathrm{IC}_{50}=375 \pm 33.4 \mu \mathrm{M}\right)$ ), since the compounds carrying a 5-membered succinimide dioxime in their structure were devoid of any activity (compounds II and 8). Further, the carbamate functionality appended to the 6-membered heterocycle enhances the $\mathrm{Fe}(\mathrm{II})$-chelating properties (compound $3\left(\mathrm{IC}_{50}=268 \pm 37.7 \mu \mathrm{M}\right)$ ). Interestingly, the free oxime $=\mathrm{N}-\mathrm{OH}$ groups do not seem to be important for complexing $\mathrm{Fe}(\mathrm{II})$, since the diacetylation of those groups led only to a minor drop in activity (compound $4\left(\mathrm{IC}_{50}=375 \pm 33.4 \mu \mathrm{M}\right)$ ). Moreover, the acyclic analogue of 3, compound 5, retains some of the Fe(II)-chelating ability ( $\left.\mathrm{IC}_{50}=671 \pm 30.7 \mu \mathrm{M}\right)$, however the latter is significantly reduced when compared to that of the parent compound. The acylation of the free amine, however, resulted in compound 10, which exhibits weak iron(II)-chelating activity $\left(\mathrm{IC}_{50}=895\right.$ $\pm 33.1 \mu \mathrm{M})$.

\subsection{Evaluation of Copper and Iron Cycling Inhibition in Ascorbate Redox System Assay}

Selected compounds ( $\mathbf{3}, \mathbf{I}$ and II) were then evaluated for their anti-Fenton properties. To shed some light on the mechanism by which these compounds can inhibit Fenton activity, their ability to halt copper and iron cycling via metal complexation was assessed in the ascorbate redox system assay [29-31]. Fenton chemistry is characterized by the oxidation of redox-active metal ions in their reduced form (e.g., $\mathrm{Fe}(\mathrm{II})$ or $\mathrm{Cu}(\mathrm{I})$ ) with hydrogen peroxide to generate hydroxyl radicals. The production of hydroxyl radicals $\left(\mathrm{HO}^{\bullet}\right)$ by metal redox cycling in the presence of oxygen and ascorbate (shown in Figure 3) was quantified by measuring the increase in fluorescence. The latter is a result of CCA, which generates fluorescent 7-hydroxy-CCA in the presence of hydroxyl radicals.

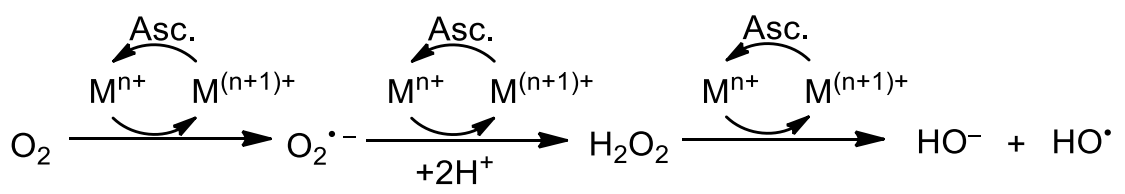

Figure 3. Production of hydroxyl radicals $\left(\mathrm{HO}^{\bullet}\right)$ by metal redox cycling in the presence of oxygen and ascorbate (Asc.). Metal ion $\left(\mathrm{M}^{\mathrm{n}+}\right)$ corresponds to $\mathrm{Cu}^{+}$or $\mathrm{Fe}^{2+}$. 
As shown in Figure 4, the fluorescence of 7-hydroxy-CCA in the copper-ascorbate system increases steadily with time and reaches a plateau after approximately 10 minutes. When compounds $\mathbf{3}$, I or II were co-incubated with the copper-ascorbate system, this process was substantially slowed down (also shown in Supporting information File 1; Figures S1-S3). As the redox-active copper ions have been related to the production of ROS, this indicates that all tested ligands were able to halt the copper redox cycling by metal complexation. Compounds 3 and I, carrying a glutarimide dioxime motif in their structures, exhibited the strongest inhibitory capacity, while compound II incorporating a succinimide dioxime moiety in its structure exhibited only a moderate inhibition of copper redox cycling. Incidentally, I has previously been demonstrated to form a strong complex with $\mathrm{Cu}$ (II) [3]. The fluorescence of 7-hydroxy-CCA in the iron-ascorbate system increases much slower with time (Figure 5) compared to copper-ascorbate system. Fe-EDTA system was employed as positive control to promote the Fenton reaction [14]. In agreement with previous findings, EDTA complexation of iron considerably increased the production rate of hydroxyl radicals (shown in Supporting information File 1; Figures S4, S6 and S8). On the other hand, co-incubation of reaction mixture with compounds I or II had practically no effect on the hydroxyl production rate (shown in Supporting information File 1; Figures S7 and S9). This was surprising, given the fact that I has previously been shown to strongly bind Fe(III) [3]. However, when compound 3 was co-incubated with the iron-ascorbate system, the production of hydroxyl radicals was halted entirely (Figure 5 and Figure S5). These findings suggest an important role of iron(II) chelation in the anti-Fenton activity of compound 3.

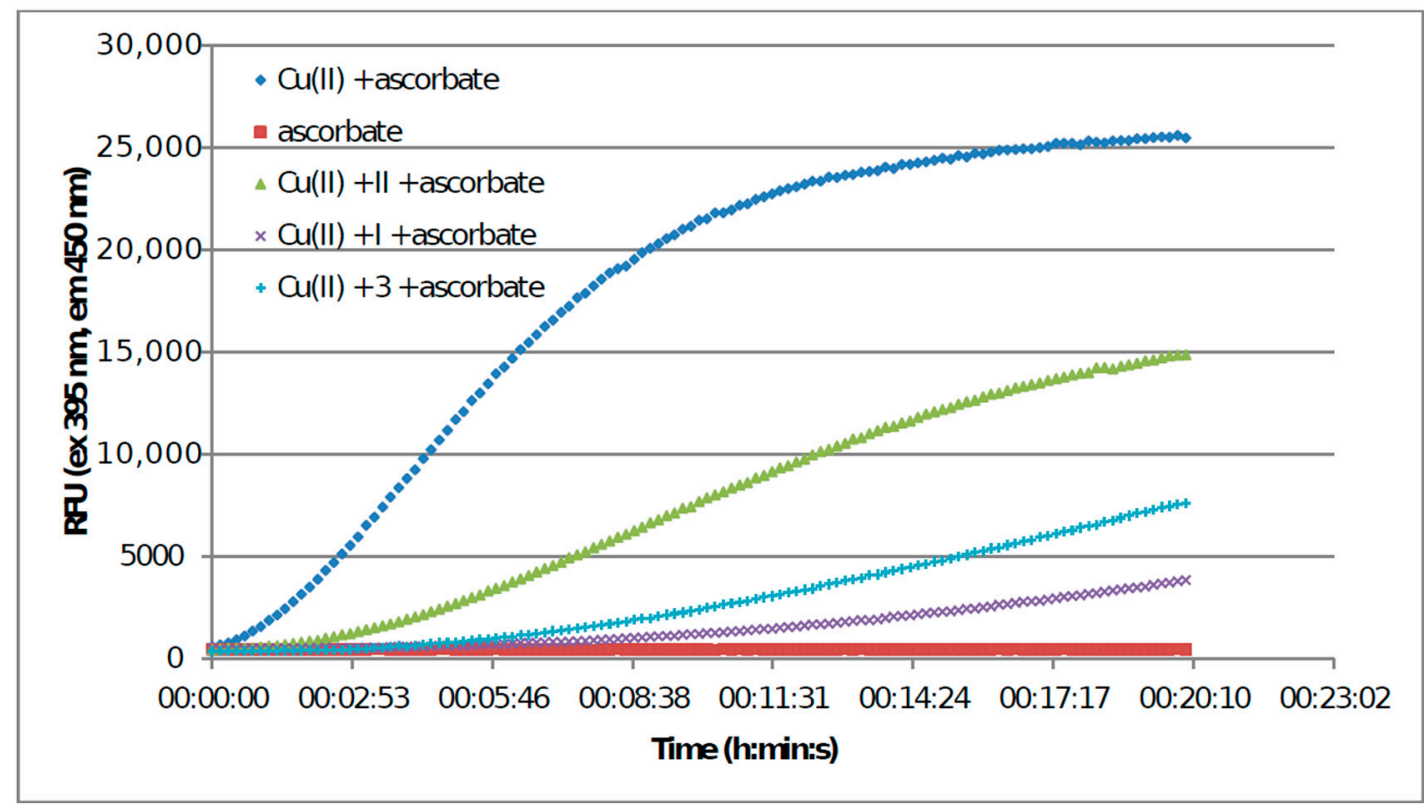

Figure 4. Fluorescence intensity of 7-hydroxy-CCA after incubation of CCA [100 $\mu \mathrm{M}]$ and $\mathrm{Cu}(\mathrm{II})$ $[10 \mu \mathrm{M}]$ with ascorbate $[300 \mu \mathrm{M}](\diamond)$. Compound II $(\Delta)[30 \mu \mathrm{M}], \mathbf{I}(\times)[30 \mu \mathrm{M}]$ or $3(+)[30 \mu \mathrm{M}]$ were added $30 \mathrm{~min}$ prior to ascorbate. Ascorbate (घ) without $\mathrm{Cu}(\mathrm{II})$ was a negative control. All solutions except $\mathrm{CuSO}_{4}$ (dissolved in Milli-Q water only) were prepared in $\mathrm{KH}_{2} \mathrm{PO}_{4}$ [20 $\mu \mathrm{M}$ ], $\mathrm{NaCl}[100 \mu \mathrm{M}]$ buffer containing desferryl $[1 \mu \mathrm{M}], \mathrm{pH}=7.4$. 


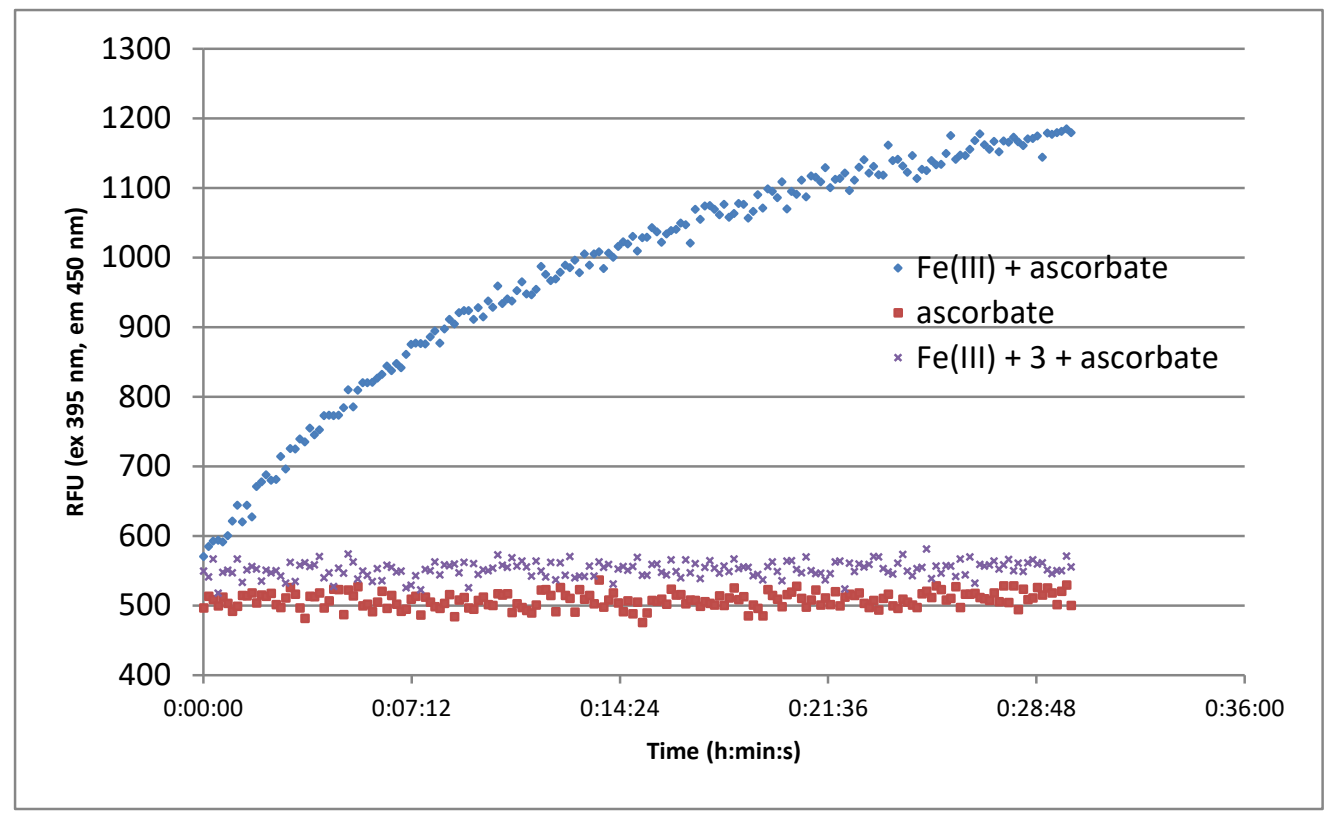

Figure 5. Fluorescence intensity of 7-hydroxy-CCA after incubation of CCA [100 $\mu \mathrm{M}]$ and Fe(III) $[10 \mu \mathrm{M}]$ with ascorbate $[300 \mu \mathrm{M}](\triangleleft)$. Compound $3(\times)[30 \mu \mathrm{M}]$ was added $30 \mathrm{~min}$ prior to ascorbate. Ascorbate ( $\square$ ) is a negative control with CCA, buffer, 3 and ascorbate without $\mathrm{Fe}(\mathrm{III})$. All solutions except $\mathrm{FeCl}_{3}$ (dissolved in Milli-Q water only) were prepared in $\mathrm{KH}_{2} \mathrm{PO}_{4}$ [20 $\mu \mathrm{M}$ ], $\mathrm{NaCl}[100 \mu \mathrm{M}]$ buffer containing desferryl $[1 \mu \mathrm{M}], \mathrm{pH}=7.4$.

\section{Conclusions}

In conclusion, we have designed a novel route for preparing chiral cyclic imide dioximes starting from the readily available amino acids. The synthesized compounds constitute building blocks capable of being integrated into larger structures. Their iron(II)-chelating abilities have also been determined. The glutarimide dioxime moiety has been established as an iron(II)-binding motif and imparts potent antioxidant properties to the compounds. Compound $\mathbf{3}$ was shown to strongly suppress hydroxyl radical formation by preventing iron cycling via Fe-complexation. These findings provide valuable insights into the structural requirements for achieving anti-Fenton activity and highlight the potential use of glutarimide dioximes as antioxidants.

Supplementary Materials: Supplementary data related to this article are available online at http://www.mdpi. com/2076-3921/8/10/473/s1; it contains ${ }^{13} \mathrm{C}$ NMR data for all new compounds and measurements of ferrous chelating ability $\left(\mathrm{IC}_{50}\right)$ and ascorbate studies. Figure S1: Effect of compound 3 on copper redox cycling, Figure S2: Effect of compound I on copper redox cycling, Figure S3: Effect of compound II on copper redox cycling, Figure S4: Effects of compound 3 and EDTA on iron redox cycling, Figure S5: Effect of compound 3 on iron redox cycling, Figure S6: Effects of compound I and EDTA on iron redox cycling, Figure S7: Effect of compound I on iron redox cycling, Figure S8: Effects of compound II and EDTA on iron redox cycling, Figure S9: Effect of compound II on iron redox cycling.

Author Contributions: Conceptualization, J.M. and Ž.J.; methodology, J.M. and Ž.J.; writing一original draft preparation, Ž.J.; writing—review and editing, Ž.J.; visualization, J.M. and Ž.J.; supervision, Ž.J.

Funding: This work was funded by the Slovenian Research Agency (Grant No. P1-0208).

Acknowledgments: The authors thank Roger Pain for critical reading of the manuscript.

Conflicts of Interest: The authors declare no conflict of interest.

\section{References}

1. Elvidge, J.A.; Linstead, R.P.; Salaman, A.M. 37. Heterocyclic imines and amines. Part IX. Glutarimidine and the imidine from $\alpha$-phenylglutaronitrile. J. Chem. Soc. 1959, 208-215. [CrossRef] 
2. Elvidge, J.A.; Linstead, R.P. Heterocyclic imines and amines. Part III. Succinimidine. J. Chem. Soc. 1954, 442-448. [CrossRef]

3. Sun, X.; Xu, C.; Tian, G.; Rao, L. Complexation of glutarimidedioxime with Fe(III), $\mathrm{Cu}(\mathrm{II}), \mathrm{Pb}(\mathrm{II})$, and Ni(II), the competing ions for the sequestration of U(VI) from seawater. Dalton Trans. 2013, 42, 14621-14627. [CrossRef]

4. Xie, X.; Tian, Y.; Qin, Z.; Yu, Q.; Wei, H.; Wang, D.; Li, X.; Wang, X. Complexation of manganese with glutarimidedioxime: Implication for extraction uranium from seawater. Sci. Rep. 2017, 7, 43503. [CrossRef] [PubMed]

5. Buscarons, F.; Iturriaga, H. Reactivity and possible analytical application of imidodioximes. An. Real. Soc. Espan. Fis. Quim. Serie B Quim. 1967, 63, 95-100.

6. Buscarons, F.; Abello, J. On the reactivity and possible analytical application of imido-dioximes. I. Phthalimido-dioxime (1,3-dihydroximino isoindoline) and succinimido-dioxime (2,5-dihydroximino pyrrolidine). An. Real. Soc. Espan. Fis. Quim. Serie B Quim. 1962, 58, 591-600.

7. Mustapha, A.M.; Pasilis, S.P. Probing Uranyl (VI) Speciation in the Presence of Amidoxime Ligands Using Electrospray Ionization Mass Spectrometry. Rapid Commun. Mass Spectrom. 2013, 27, 2135-2142. [CrossRef] [PubMed]

8. Kang, S.O.; Vukovic, S.; Custelcean, R.; Hay, B.P. Cyclic Imide Dioximes: Formation and Hydrolytic Stability. Ind. Eng. Chem. Res. 2012, 51, 6619-6624. [CrossRef]

9. Kobuke, Y.; Tanaka, H.; Ogoshi, H. Imidedioxime as a Significant Component in So-Called Amidoxime Resin for Uranyl Adsorption from Seawater. Polym. J. 1990, 22, 179-182. [CrossRef]

10. Kusakabe, K.; Goto, A.; Morooka, S. Kinetics of Uranium Adsorption from Seawater with Imidedioxime Adsorbent. Sci. Technol. 1994, 29, 1567-1577. [CrossRef]

11. Tian, G.; Teat, S.J.; Zhang, Z.; Rao, L. Sequestering uranium from seawater: Binding strength and modes of uranyl complexes with glutarimidedioxime. Dalton Trans. 2012, 41, 11579-11586. [CrossRef] [PubMed]

12. Grant, C.D.; Kang, S.O.; Hay, B.P. Synthesis of a hydrophilic naphthalimidedioxime. J. Org. Chem. 2013, 78, 7735-7740. [CrossRef] [PubMed]

13. Thangavel, A.; Ramasamy, S.; Arunagiri, M.; Marimuthu, S.A.; Dhanabal, S.; Gurusamy, S.K.; Nachiappan, Y. A Process for Preparation of 4,4'-(1-Methyl-1,2-Ethandiyl)-bis-(2,6-Piperazine-Dione). WO 2012081036 A2, 21 June 2012.

14. Guo, M.; Perez, C.; Wei, Y.; Rapoza, E.; Su, G.; Bou-Abdallah, F.; Chasteen, N.D. Iron-binding properties of plant phenolics and cranberry's bio-effects. Dalton Trans. 2007, 43, 4951-4961. [CrossRef] [PubMed]

15. Zhou, T.; Ma, Y.; Kong, X.; Hider, R.C. Design of iron chelators with therapeutic application. Dalton Trans. 2012, 41, 6371-6389. [CrossRef] [PubMed]

16. Leopoldini, M.; Russo, N.; Chiodo, S.; Toscano, M. Iron chelation by the powerful antioxidant flavonoid quercetin. J. Agric. Food Chem. 2006, 54, 6343-6351. [CrossRef] [PubMed]

17. Heli, H.; Mirtorabi, S.; Karimian, K. Advances in iron chelation: An update. Expert Opin. Ther. Patents 2011, 21, 819-856. [CrossRef] [PubMed]

18. Phansi, P.; Henríquez, C.; Palacio, E.; Wilariat, P.; Nacapricha, D.; Cerdà, V. An automated catalytic spectrophotometric method for manganese analysis using a chip-multisyringe flow injection system (Chip-MSFIA). Anal. Methods 2014, 6, 5088-5096. [CrossRef]

19. Jeitner, T.M. Optimized ferrozine-based assay for dissolved iron. Anal. Biochem. 2014, 454, 36-37. [CrossRef]

20. Green, K.N.; Lincoln, K.M.; Gonzalez, P. Antioxidant Small Molecules Aimed at Targeting Metal-Based Oxidative Stress in Neurodegenerative Disorders. U.S. Patent 8,969,548, 3 March 2015.

21. Lioux, T.; Busson, R.; Rozenski, J.; Nguyen-Distèche, M.; Frère, J.-M.; Herdewijn, P. Synthesis of Peptidoglycan Units with UDP at the Anomeric Position. Collect. Czech. Chem. Commun. 2005, 70, 1615-1641. [CrossRef]

22. Pozdnev, V.F. Activation of carboxylic acids by pyrocarbonates. Application of di-tert-butyl pyrocarbonate as condensing reagent in the synthesis of amides of protected amino acids and peptides. Tetrahedron Lett. 1995, 36, 7115-7118. [CrossRef]

23. Maetz, P.; Rodriguez, M. A simple preparation of N-protected chiral $\alpha$-aminonitriles from N-protected $\alpha$-amino acid amides. Tetrahedron Lett. 1997, 38, 4221-4222. [CrossRef]

24. Jakopin, Ž.; Roškar, R.; Sollner Dolenc, M. Synthesis of 3,5-disubstituted 1,2,4-oxadiazoles as peptidomimetic building blocks. Tetrahedron Lett. 2007, 48, 1465-1468. [CrossRef] 
25. Yeung, M.C.; Klein, L.L.; Flentge, C.A.; Randolph, J.T.; Zhao, C.; Sun, M.; Dekhtyar, T.; Stoll, V.S.; Kempf, D.J. Oximinoarylsulfonamides as potent HIV protease inhibitors. Bioorg. Med. Chem. Lett. 2005, 15, 2275-2278. [CrossRef] [PubMed]

26. Jakopin, Ž. The design and synthesis of Ala-Glu/iGln mimetics: Heterocyclic building blocks for pseudopeptides. Tetrahedron Lett. 2015, 56, 504-506. [CrossRef]

27. Gülçin, I. Antioxidant activity of caffeic acid (3,4-dihydroxycinnamic acid). Toxicology 2006, 217, $213-220$. [CrossRef] [PubMed]

28. Ak, T.; Gülçin, I. Antioxidant and radical scavenging properties of curcumin. Chem. Biol. Interact. 2008, 174, 27-37. [CrossRef]

29. Guilloreau, L.; Combalbert, S.; Sournia-Saquet, A.; Mazarguil, H.; Faller, P. Redox chemistry of copper-amyloid- $\beta$ : The generation of hydroxyl radical in the presence of ascorbate is linked to redox-potentials and aggregation state. ChemBioChem 2007, 8, 1317-1325. [CrossRef]

30. Lincoln, K.M.; Richardson, T.E.; Rutter, L.; Gonzalez, P.; Simpkins, J.W.; Green, K.N. An N-Heterocyclic Amine Chelate Capable of Antioxidant Capacity and Amyloid Disaggregation. ACS Chem. Neurosci. 2012, 3, 919-927. [CrossRef]

31. Mao, F.; Yan, J.; Li, J.; Jia, X.; Miao, H.; Sun, Y.; Huang, L.; Li, X. New multi-target-directed small molecules against Alzheimer's disease: A combination of resveratrol and clioquinol. Org. Biomol. Chem. 2014, 12, 5936-5944. [CrossRef]

(C) 2019 by the authors. Licensee MDPI, Basel, Switzerland. This article is an open access article distributed under the terms and conditions of the Creative Commons Attribution (CC BY) license (http://creativecommons.org/licenses/by/4.0/). 